\title{
Encapsulating and Manipulating Component Object Graphics (COGs) using SVG
}

\author{
Alexander J. Macdonald \\ Document Engineering Laboratory \\ School of Computer Science \\ University of Nottingham \\ Nottingham, NG8 1BB, UK \\ ajm@cs.nott.ac.uk
}

\author{
David F. Brailsford \\ Document Engineering Laboratory \\ School of Computer Science \\ University of Nottingham \\ Nottingham, NG8 1BB, UK \\ dfb@cs.nott.ac.uk
}

\author{
Steven R. Bagley \\ Document Engineering Laboratory \\ School of Computer Science \\ University of Nottingham \\ Nottingham, NG8 1BB, UK \\ srb@cs.nott.ac.uk
}

\begin{abstract}
Scalable Vector Graphics (SVG) has an imaging model similar to that of PostScript and PDF but the XML basis of SVG allows it to participate fully, via namespaces, in generalised XML documents.

There is increasing interest in using SVG as a Page Description Language and we examine ways in which SVG document components can be encapsulated in contexts where SVG will be used as a rendering technology for conventional page printing.

Our aim is to encapsulate portions of SVG content (SVG COGs) so that the COGs are mutually independent and can be moved around a page, while maintaining invariant graphic properties and with guaranteed freedom from side effects and mutual interference. Parellels are drawn between COG implementation within SVG's tree-based inheritance mechanisms and an earlier COG implementation using PDF.
\end{abstract}

\section{Categories and Subject Descriptors}

E.1 [Data]: Data Structures - Trees; I.7.2 [Document and Text Processing]: Document Preparation — Markup Languages; I.7.4 [Document and Text Processing]: Electronic Publishing.

General Terms: Algorithms, Documentation.

\section{Keywords}

XML, SVG, PDF, parameterization, component object graphics

\section{INTRODUCTION}

The World Wide Web Consortium (W3C), set up a working group in 1998, to draw up draft proposals for Scalable Vector Graphics (SVG) in response to a need for better rendering of material such as line diagrams, schematics, and maps. At present there is only limited native support within Web browsers for rendering SVGfor the most part popular browsers such as Internet Explorer need to install an SVG plug-in supplied by Adobe Systems Inc. Although the syntax of SVG is XML based, the semantics of its graphics model are similar to those of PostScript and PDF. In common with these two languages, SVG combines graphical sophistication with the ability to place text strings accurately.

Currently, with SVG 1.1, the major use is for "vector graphic inserts' into conventional Web pages. But with SVG 1.2 close to approval there is now the prospect of SVG acquiring a page model (via pagesets [1]). This, in turn, means that SVG could become a

FINAL DRAFT of paper accepted for:

DocEng'05 November 2-4, 2005, Bristol, United Kingdom

Copyright 2005 Macdonald, Brailsford and Bagley viable rendering technology for a Page Description Language (PDL) for conventional hard-copy printing. Danilo and Fujisawa [2] have already surveyed some of the problems, ranging from frame buffer size to filters and ICC colour spaces, which need to be addressed before SVG can become a satisfactory PDL. Our concerns run even deeper: we want to develop ways to determine the graphical state at the root of a given SVG subtree sufficiently accurately, and to encapsulate it in some way, so that the subtree can then be extracted and used elsewhere.

\subsection{Previous work and present aims}

In a previous paper [3] we developed the idea of Component Object Graphics (COGs) in which encapsulated graphic objects can have their page positions and certain needed resources (e.g. fonts) declared at the head of a suitable data structure. Our initial implementation of COGs was in PDF and the PDF data structure we used for encapsulation was the FormXobject.

In the Component-Object Graphic model the pages in a PDL are no longer described as monolithic page streams (where the effect of each operator depends on the operators that have been executed before it) but rather as independent graphical objects, or COGs. A COG is a self-contained block that describes how to draw itself in a manner independent of any other COG appearing before it, while ensuring also that it does not affect the appearance of any COG imaged after it. In other words, adding or removing a COG from a page should have no visible side-effect on any other COG on that page.

A key advantage of PDF COGs is that a single COG instance can be shared throughout a document. Our COG model allows us to encapsulate PDF inside a FormXObject structure while the PDF graphical system (and its capability for saving and restoring graphic state) make it possible to establish a relative co-ordinate system within the COG and to make clear at the head of the FormXObject dictionary the resources the COG will use. The sequence of COGs on a page is invoked by executing the corresponding FormXObjects using the PDF Do operator.

The COG model is by no means limited to PDF. It is potentially applicable to any PDL that offers the possibility of encapsulation and re-use of material. So, if SVG 1.2 will truly be a future alternative to PDF we wanted to find out whether SVG COGs were feasible and, if so, how the tree-based inheritance mechanisms for graphic properties might lead to new problems and opportunities as compared to the more conventional document-based and page-based inheritance properties of PDF.

We now look at ways in which SVG COGs can be defined, manipulated on the page and made shareable. 


\subsection{Graphical independence of SVG COGs}

By using the previously mentioned $\langle g>$ element it is very easy to group together all of the graphical content of a COG. However, SVG 1.2 introduces the ref() matrix, which computes the inverse of the current viewport's transformation matrix. Thus if the SVG code is left unchecked it could effectively break out of the COG nature of the document and position itself using absolute page co-ordinates rather than relative to the origin of its parent. This can be prevented by additionally wrapping the COGs inside <svg > elements so that they each exist as a separate document fragment. This also means COGs are explicitly clipped to their bounding box, and so more closely match the behaviour of PDF COGs. Another benefit is that resources such as gradients and embedded fonts can be stored in the COG.

Because all positioning in SVG is done relative to the origin of the current coordinate system we can apply a transformation, to the group as a whole, which will alter that system. This allows the entire COG to be moved, scaled or rotated in any way whilst preserving the internal positioning of the contents of the COG. To demonstrate the graphical independence of the SVG COGs an ECMAScript was created that allows the user to move, scale and rotate any of the COGs in a document. In effect it mimics the effect of the PDF COG manipulation program (written in $\mathrm{C}++$ ) that was described in [3]. It should be noted that while the Acrobat plug-in extends the Acrobat viewer (and so is available to all COG PDF documents) the ECMAScript must be included, or linked to, in the COG SVG document before it can be used.

It was encouraging to find that the ECMAscript SVG COG manipulator worked correctly and, as a given COG is moved around the page, all other COGs on the page are unaffected. This is demonstrated in Figure 3.6 where the ECMAscript COG manipulator has been used to shift the "OTHERS" COG in the Helvetica House menu. Also visible are shaded squares, which are controls to switch between translation, rotation and scaling of the selected COG.

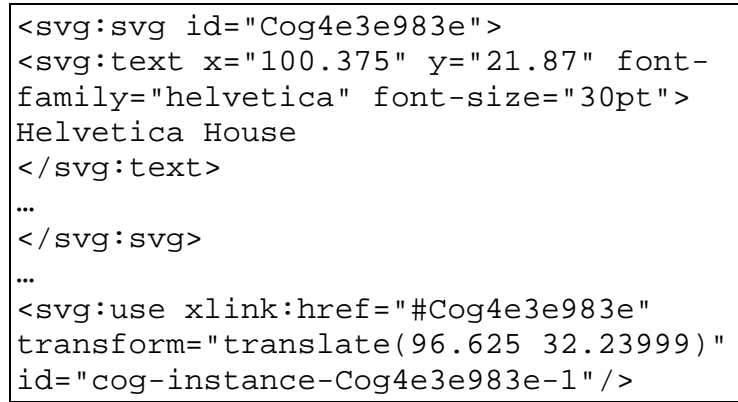

Figure 3.5 - An SVG COG definition and a reference to it.

\subsection{Creating SVG COGs}

In addition to hand-coding SVG COGs we are presently in the process of converting several of our XSLT-based SVG codegeneration facilities into a form such that they can generate SVG COGs. A further useful source of SVG COGs is also available to us because it has proved possible to modify Mong's PDF-to-SVG converter [4] so that it converts PDF COGs into SVG COGs.

\section{CONCLUSIONS AND FUTURE WORK}

All the effects of PDF COGs described in [3] have been successfully replicated in SVG. The use of $\langle\operatorname{def} \mathrm{s}\rangle$ and $\langle\mathrm{svg}\rangle$ to encapsulate SVG code parallels the use of a FormXObject structure in PDF whereas SVG's <use> operator parallel's PDF's execution of a FormXObject via Do.

Note carefully that the simple SVG COGs we have created are

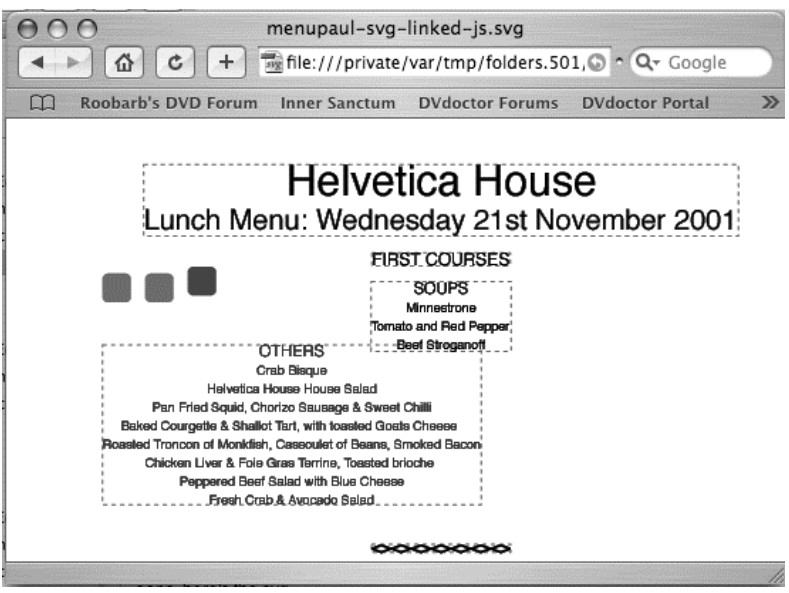

\section{Figure 3.6 - ECMAScript demonstration of the graphical independence of SVG COGs}

just like their PDF counterparts in being invariant in their appearance wherever they might be invoked. This behaviour is a consequence of the fact that PDF is a final-form PDL, with no facilities for parameterised late-bound adjustments to appearance.

By contrast, SVG is not a final-form PDL. It is parameterisable via XML attributes (see for example the <text $>$ node in Figure 3.4). If a more powerful mechanism for parameter forwarding and binding can be devised one might achieve parameterised COGs. An example would be the insertion of formal parameters inside text strings to enable late-bound items such as the price of an item, or the start and finish dates of a special offer, to be substituted at the last possible moment, just before the page is rendered and printed. Work continues on exploring this exciting possibility.

\section{ACKNOWLEDGEMENTS}

Thanks are due to Hewlett Packard (UK) and EPSRC for supporting Alex Macdonald's PhD studentship. In particular we thank John Lumley and Tony Wiley of HP (UK) for technical insights and administrative help. Thanks are also due to Jon Ferraiolo of Adobe Systems Inc for information on SVG 1.2.

\section{REFERENCES}

[1] SVG 1.2 - Multiple Pages. http://www.w3.org/TR/2004/WDSVG12-20041027/multipage.html

[2] Alex Danilo and Jun Fujisawa, "SVG as a Page Description Language" http://www.svgopen.org/2002/papers

[3] Steven Bagley, David Brailsford, and Matthew Hardy, "Creating reusable well-structured PDF as a sequence of Component Object Graphic (COG) elements.,' in Proceedings of the ACM Symposium on Document Engineering (DocEng'03), pp. 58-67, ACM Press, 20-22 November 2003.

[4] Julius Mong and David Brailsford, "Some experiments in using SVG as the rendering model for structured and graphically complex Web material,'” in Proceedings of the ACM Symposium on Document Engineering (DocEng'03), pp. 88-91, ACM Press, 20-22 November 2003. 